\title{
A UNICELLULAR UNIVERSAL QUASINILPOTENT WEIGHTED SHIFT
}

\author{
DOMINGO A. HERRERO
}

(Communicated by Palle E. T. Jorgensen)

\begin{abstract}
For a suitably chosen sequence of weights $\left\{\alpha_{n}\right\}$, the unilateral weighted shift $Q$ on $l^{p} \quad(1 \leq p<\infty)$, defined by $Q e_{n}=\alpha_{n} e_{n+1} \quad(n \geq 1)$, is a unicellular quasinilpotent operator such that $Q^{k}$ is not compact for any power $k \geq 1$. As a corollary, several applications to approximation of Hilbert space operators are given.
\end{abstract}

Inductively, define $\gamma_{1}=1, \gamma_{2}=\frac{1}{4}, \gamma_{n}=\left(\gamma_{1} \gamma_{2} \cdots \gamma_{n-1}\right)^{n}$, and let $\left\{\alpha_{n}\right\}$ be the sequence

$$
\gamma_{1}, \gamma_{2}, \ldots, \gamma_{9}, \gamma_{1}, \gamma_{2}, \ldots, \gamma_{90}, \gamma_{1}, \gamma_{2}, \ldots, \gamma_{900}, \gamma_{1}, \gamma_{2}, \ldots, \gamma_{9,000}, \gamma_{1}, \ldots
$$

Let $Q$ be the unilateral weighted shift defined by $Q e_{n}=\alpha_{n} e_{n+1} \quad(n \geq 1)$ with respect to the canonical basis $\left\{e_{n}\right\}_{n \geq 1}$ of $l^{p} \quad(1 \leq p<\infty)$, and let $Q_{r}$ be similarly defined, with the first $10^{r}$ weights replaced by zeros. If $10^{r-1} \leq k<$ $10^{r}$, then a straightforward calculation shows that

$$
\left\|\left(Q_{r}\right)^{k}\right\|=\text { essential } \operatorname{norm}\left(Q_{r}\right)^{k}=\prod_{j=1}^{k} \gamma_{j}>0
$$

(so that $\left(Q_{r}\right)^{k}$ is not a compact operator), and therefore the spectral radius of $Q_{r}$ cannot exceed

$$
\left(\prod_{j=1}^{k} \gamma_{j}\right)^{1 / k}<\left(\gamma_{k}\right)^{1 / k} \rightarrow 0 \quad(k \rightarrow \infty) .
$$

Since $Q-Q_{r}$ has finite rank and the spectrum of a unilateral weighted shift is always connected, we conclude that

$Q$ is quasinilpotent and $Q^{k}$ is not compact for any $k \geq 1$.

(The reader is referred to the monograph [13] for general information about unilateral weighted shifts.)

Received by the editors April 28, 1989 and, in revised form, November 7, 1989.

1980 Mathematics Subject Classification (1985 Revision). Primary 47A15, 47B37, 47B99.

Key words and phrases. Universal quasinilpotent, unilateral weighted shift, strictly cyclic operator, unicellular operator.

This research was partially supported by a grant of the National Science Foundation. 
It only remains to show that $Q$ is unicellular, that is, the invariant subspace lattice of $Q$ is the chain

$$
\{0\}, \mathscr{M}_{t}=\bigvee\left\{e_{n}\right\}_{n \geq t} \quad\left(t \geq 1 ; \mathscr{M}_{1}=l^{p}\right)
$$

If $1 \leq m \leq[n / 2]$ and $n-m+1 \leq 10^{r}<n$, then $n-m+s=10^{r}$ for some $s(1 \leq s \leq m-1)$ and

$$
\begin{aligned}
\prod_{j=1}^{m} \frac{\alpha_{n-j+1}}{\alpha_{j}} & =\left(\frac{\alpha_{n-m+s+1} \alpha_{n-m+s+2} \cdots \alpha_{n}}{\alpha_{1} \alpha_{2} \cdots \alpha_{m-s}}\right)\left(\frac{\alpha_{n-m+1} \alpha_{n-m+2} \cdots \alpha_{n-m+s}}{\alpha_{m-s+1} \alpha_{m-s+2} \cdots \alpha_{m}}\right) \\
& \leq \frac{\alpha_{n-m+1} \alpha_{n-m+2} \cdots \alpha_{n-m+s}}{\alpha_{m-s+1} \alpha_{m-s+2} \cdots \alpha_{m}} \leq\left(\gamma_{10^{r}-10^{r-1}}\right)^{1-1 /\left(10^{r}-10^{r-1}\right)} \leq 4^{-m}
\end{aligned}
$$

because $m<n-m+s$.

If $1 \leq m \leq[n / 2]$ and $10^{r}<n-m+1<n \leq 10^{r+1}$, then

$$
\prod_{j=1}^{m} \frac{\alpha_{n-j+1}}{\alpha_{j}} \leq \begin{cases}1, & \text { if } m \leq 10 \\ 4^{10-m}, & \text { if } m>10\end{cases}
$$

Thus, we have

$$
\begin{aligned}
\sup _{n} \sum_{m=0}^{n} \prod_{j=1}^{m} \frac{\alpha_{n-j+1}}{\alpha_{j}} & \leq 2+2 \sup _{n} \sum_{m=1}^{[n / 2]} \prod_{j=1}^{m} \frac{\alpha_{n-j+1}}{\alpha_{j}} \\
& <2+2 \cdot 4^{10} \sum_{m=1}^{\infty} 4^{-m}<4^{10}<\infty
\end{aligned}
$$

It follows from [4, 11] (or [12]) that $Q$ is a strictly cyclic operator; that is, $l^{p}=\mathscr{A}(Q) e_{1}$, where $\mathscr{A}(Q)$ is the uniform closure of the polynomials in $Q$ and $I$. Thus, $\mathscr{A}(Q)$ is a maximal abelian Banach algebra of operators (acting on $l^{p}$ ), whose Gelfand spectrum coincides with $\sigma(Q)=\{0\} \quad$ [7, Theorem 4]. It readily follows that $\mathscr{A}(Q)$ has exactly one maximal ideal $\mathscr{A}_{1}(Q)$. Under the isomorphism of Banach spaces defined by $A \rightarrow A e_{1}$ (from $\mathscr{A}(Q)$ onto $l^{p}$ ), (closed) ideals of $\mathscr{A}(Q)$ correspond to invariant subspaces of $Q$; in particular, $\mathscr{A}_{1}(Q) e_{1}=\mathscr{M}_{2}$ includes all the nontrivial invariant subspaces of $Q$.

Observe that $Q \mid \mathscr{M}_{t}$ is a quasinilpotent unilateral weighted shift with weight sequence $\left\{\alpha_{n}^{(t)}=\alpha_{n+t}\right\}_{n \geq 1}$. A cumbersome calculation (similar to the above one) shows that

$$
\sup _{n} \sum_{m=0}^{n} \prod_{j=1}^{m} \frac{\alpha_{n-j+1}^{(t)}}{\alpha_{j}^{(t)}} \leq C_{t}<\infty,
$$

hence we conclude that every nontrivial invariant subspace of $Q \mid \mathscr{M}_{t}$ is a subspace of $\mathscr{M}_{t+1}(t \geq 1)$.

It readily follows that

$$
\text { Lat } Q=\left\{\{0\} ; \mathscr{M}_{t}, t \geq 1\right\} \text {. }
$$

The existence of these kinds of operators affirmatively answers a question of J. B. Conway (personal communication). 
1. The above argument (if $Q \mid \mathscr{M}_{t}$ is strictly cyclic for all $t \geq 1$, then $Q$ is unicellular) is due to R. Gellar [5].

2. For $1<p<\infty$, the adjoint of $Q$ is the backward weighted shift $R$, defined by $R e_{1}=0, R e_{n}=\alpha_{n-1} e_{n-1}\left(n>1 ; R\right.$ acts on $l^{q}$, where $\left.\frac{1}{p}+\frac{1}{q}=1\right)$. Clearly,

$$
\text { Lat } R=\left\{\{0\} ; \bigvee\left\{e_{n}\right\}_{n=1}^{t}, t \geq 1 ; l^{q}\right\} \text {. }
$$

3. In the Hilbert space case $(p=2), Q$ and $R$ are "universal quasinilpotents"; that is, the closure of the similarity orbit of $Q$ (or $R$ ) coincides with the set of limits of nilpotent operators; more precisely,

$\mathscr{S}(Q)^{-}=\mathscr{S}(R)^{-}=\left\{A \in \mathscr{L}\left(l^{2}\right):\right.$ the spectrum $\sigma(A)$ and the essential spectrum $\sigma_{e}(A)$, of $A$, are connected sets containing the origin, and $\operatorname{ind}(\lambda-A)=0$ for each complex $\lambda$ such that $\lambda-A$ is a semi-Fredholm operator $\}$

(see $[1,2,5,6]$ or $\left[7\right.$, Chapter 8]). (Here $\mathscr{S}(T):=\left\{W T W^{-1}: W\right.$ is invertible $\}$ denotes the similarity orbit of the operator $T$, and the upper bar indicates norm-closure.)

By using this last observation, several known results in the literature can be given very simple proofs. Two examples:

Corollary 1 (D. W. Hadwin [6]). The norm-closure of the set of all unicellular operators on a Hilbert space $\mathscr{H}$ coincides with

$$
\begin{aligned}
& \left\{A \in \mathscr{L}(\mathscr{H}): \sigma(A) \text { and } \sigma_{e}(A)\right. \text { are connected sets and } \\
& \operatorname{ind}(\lambda-A)=0 \text { for all } \lambda \in \mathbb{C} \text { such that } \lambda-A \text { is semi-Fredholm }\} .
\end{aligned}
$$

Proof. Let $\mathscr{W}$ denote the above described set. The continuity properties of the Riesz-Dunford functional calculus and the stability properties of the semiFredholm operators indicate that every unicellular operator on $\mathscr{H}$ is a member of $\mathscr{W}$ (see, e.g., [10, Chapter 1]; this inclusion is valid in every Banach space). Since $\mathscr{W}$ is a closed subset of $\mathscr{L}(\mathscr{H})$, it must include the closure of the unicellular operators.

On the other hand, if $A \in \mathscr{W}$ and $\mu \in \sigma_{e}(A)$, then $A-\mu \in \mathscr{S}(Q)^{-}$. Thus,

$$
\mathscr{W}=\bigcup_{\mu \in \mathbb{C}}\left\{\mu+T: T \in \mathscr{S}(Q)^{-}\right\} .
$$

Since $Q$ is unicellular, we are done.

A subspace $\mathscr{M}$ is stably invariant for $T \in \mathscr{L}(\mathscr{H})$ if, for each $\varepsilon>0$ there exists $\delta>0$ such that if $\left\|T-T^{\prime}\right\|<\delta$, then $T^{\prime}$ has an invariant subspace $\mathscr{M}^{\prime}$ satisfying

$$
\left\|P_{\mathscr{M}}-P_{\mathscr{M}^{\prime}}\right\|<\varepsilon,
$$

where $P_{\mathscr{M}}$ denotes the orthogonal projection of $\mathscr{H}$ onto the subspace $\mathscr{M}$.

Corollary 2 (C. Apostol, C. Foias, and N. Salinas [3]; also see [2, Appendix II]). Assume that $\sigma(T)$ and $\sigma_{e}(T)$ are connected sets and $\operatorname{ind}(\lambda-T)=0$ for 
each $\lambda \in \mathbb{C}$ such that $\lambda-T$ is a semi-Fredholm operator. Then $T$ is "stably transitive"; that is, the only stably invariant subspaces of $T$ are the trivial ones $(\{0\}$ and $\mathscr{H})$.

Proof. Let $\mu \in \sigma_{e}(T)$; then

$$
T-\mu \in \mathscr{S}(Q)^{-}=\mathscr{S}(R)^{-} .
$$

Thus, for each $\delta>0$ there exist $Q^{\prime}$ similar to $Q$ and $R^{\prime}$ similar to $R$ such that

$$
\left\|T-Q^{\prime}\right\|<\delta \text { and }\left\|T-R^{\prime}\right\|<\delta .
$$

Let $\mathscr{Q}$ and $\mathscr{R}$ be any two nontrivial subspaces of $\mathscr{H}$ invariant under $Q^{\prime}$ and, respectively, under $R^{\prime}$. Since $\mathscr{Q}$ is necessarily finite-dimensional and $\mathscr{R}$ is necessarily of finite codimension, we have $\left\|P_{\mathscr{Q}}-P_{\mathscr{R}}\right\| \geq 1$. Furthermore,

for each subspace $\mathscr{M}$ of $\mathscr{H}$ !

$$
\max \left[\left\|P_{\mathscr{Q}}-P_{\mathscr{M}}\right\|,\left\|P_{\mathscr{R}}-P_{\mathscr{M}}\right\|\right] \geq 1
$$

Therefore, $T$ cannot have any nontrivial stably invariant subspace.

It is clear from this last example that the operators $Q$ and $R$ can play a very interesting role in the still open problem of characterizing all the stably invariant subspaces of a given operator $T$.

\section{REFERENCES}

1. C. Apostol, Universal quasinilpotent operators, Rev. Roumaine Math. Pures Appl. 25 (1980), 135-138.

2. C. Apostol, L. A. Fialkow, D. A. Herrero, and D. Voiculescu, Approximation of Hilbert space operators. Volume II, Res. Notes in Math., vol. 102, Pitman, Boston, London, and Melbourne, 1984.

3. C. Apostol, C. Foiaş, and N. Salinas, Stable invariant subspaces, Integral Equations Operator Theory 8 (1985), 721-750.

4. M. Embry, Strictly cyclic operator algebras on a Banach space, Pacific J. Math. 45 (1973), 443-452.

5. R. Gellar, Weighted shifts on Banach spaces, Dissertation, Columbia University, 1968.

6. D. W. Hadwin, An addendum to limsups of lats, Indiana Univ. Math. J. 29 (1980), 313319.

7. D. A. Herrero, Operator algebras of finite strict multiplicity, Indiana Univ. Math. J. 22 (1972), 13-24.

8. __ Universal quasinilpotent operators, Acta Sci. Math. (Szeged) 38 (1976), 291-300.

9. __ Almost every quasinilpotent Hilbert space operator is a universal quasinilpotent, Proc. Amer. Math. Soc. 71 (1978), 212-216.

10. __, Approximation of Hilbert space operators. Volume I, Res. Notes in Math., vol. 72, Pitman, Boston, London, and Melbourne, 1982.

11. E. Kerlin and A. Lambert, Strictly cyclic shifts on $l^{p}$, Acta Sci. Math. (Szeged) 35 (1973), 87-94.

12. N. K. Nikol'skii, The unicellularity and nonunicellularity of weighted shift operators, Dokl. Akad. Nauk SSSR 172 (1967), 91-94. (Russian)

13. A. L. Shields, Weighted shift operators and analytic function theory, Math. Surveys Monogr., vol. 13, Amer. Math. Soc., Providence, RI, 1974, pp. 49-128.

Department of Mathematics, Arizona State University, Tempe, Arizona 85287 